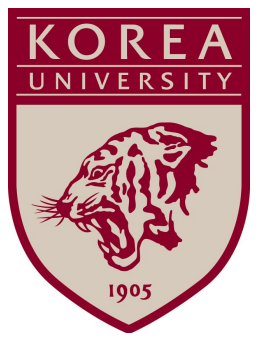

Discussion Paper Series

No. 0918

Dec 2009

\title{
The Loan Contract with Costly State Verification and Subjective Beliefs
}

\author{
Carsten Krabbe Nielsen
}

The Institute of Economic Research - Korea University

Anam-dong, Sungbuk-ku, Seoul, 136-701, South Korea, Tel: (82-2) 3290-1632, Fax: (82-2) 928-4948

Copyright (C) 2009 IER. 


\title{
The Loan Contract with Costly State Verification and Subjective Beliefs
}

\author{
by \\ Carsten Krabbe Nielsen*, Universita Cattolicá and Korea University
}

Summary We generalize the characterization of the loan contract due to Gale and Hellwig(1985) to the case of risk aversion of the borrower and diverse subjective beliefs about the outcome of the investment. We continue to assume costly state verification (Townsend, 1979) i.e. that the lender must incur costs in order to observe the outcome of the project. Contract terms now reflect returns on capital as well as risk sharing and trade on the differences in probabilities. Because there are no financial markets where agents could purchase insurance for state contingencies, private contracting replaces markets for contingent claims. This also means that verification states are not necessarily interpreted as "default" states. We characterize the optimal contract showing that (i) the contractual payoff in verification states varies by states in accord with risk aversion and probability belief of the two parties, and (ii) the verification region may consist of several intervals. We provide conditions and examples to show that when the borrower is more optimistic than the bank, there may be fewer verification regions.

Key words: Loan Contract, Costly State Verification, Subjective Beliefs

\footnotetext{
${ }^{*}$ The results presented here are the outcome of what was initially a joint study with Mordecai Kurz of Stanford University who due to other obligations was unable to participate in its completion. All results presented have been derived by the author. Suggestions from Mordecai Kurz, Maurizio Motolese, Gerd Winrich and participants in FESEM, 2005 and ESEM,2004 are greatly appreciated.
} 


\title{
The Loan Contract with Costly State Verification and Subjective Beliefs
}

\author{
by \\ Carsten Krabbe Nielsen
}

\section{Introduction}

Gale and Hellwig(1985) extended Townsend's(1979) contracting approach to debt contracts and has had a large impact on the way markets for loans are being studied. Their model is commonly explored in textbooks (e.g. Xavier and Rochet,1997, Chapter 4 and Romer, 2001, Chapter 8) and is often used as benchmark in applications (e.g. Boyd and Smith, 1994, Khalil and Parigi, 1998, and Mookherjee and Png, 1989). In addition it has become a vehicle for understanding how credit markets may affect the macro economy by magnifying shocks to the economy, a so called accelerator effect (see f.i. Bernanke and Gertler, 1989, Bernanke, Gertler and Gilchrist, 1999, and Carlstrom and Fuerst, 1997)

In this study we generalize the Gale and Hellwig model to the case where the borrower is risk averse and holds expectation different from those of the bank. We provide a relatively simple and intuitive characterization of the optimal contract under these circumstances. A predecessor to our analysis is the contribution of Carlier and Renou (2003), who showed that the so called standard debt contract is often not the outcome when expectations are diverse, something which is confirmed by our characterization.

We briefly review the basic set-up of the Gale and Hellwig (1985) model. An entrepreneur has equity capital $N$ used as collateral when seeking to borrow the amount $B$ from the bank. The amount $N+B$ is invested in a risky project whose return depends on a stochastic state, $s \in$ $S$. The return minus payments to the bank is then consumed by the entrepreneur. The standard assumptions are: (i) consumption is non-negative, (ii) both the borrower and the bank are risk neutral, (iii) the borrower and the bank hold the same probability belief about the distribution of $s$, (iv) The bank can ex-ante commit to actions ex-post (i.e. verification) and contracts are non-stochastic. The state, $s$ is ex-post asymmetrically observed: The entrepreneur can observe the realized state at no cost while the bank needs to incur an audit cost, $c(s, B+N)$. According to the contract, payments to the bank are then specified as a function of the announced state. A bank's decision to verify the state is commonly interpreted as a default by the borrower who announces that he cannot meet the contractual payment. The conclusion is a standard loan contract partitioning the set of states $S$ into two intervals. An audit interval $S^{*}$ of states of the lowest returns where the bank spends the observation cost $c$ to verify the state and an 
interval, $S \backslash S^{*}$ of high investment returns where no state verification takes place. If $s \in S^{*}$, the borrower is in default and consumes nothing while the bank receives the available resources. For $s \in S \backslash S^{*}$ the payment to the bank is a constant independent of the state and the borrower receives the balance.

Gale and Hellwig briefly considered how the contract might change if the borrower is risk averse. Garino and Simmons(2006) study in further detail the optimal contract when the borrower is risk averse but both papers assume that the borrowers and the bank hold the same probability beliefs about the state. Assuming the verification cost is independent of the state, they show that when the borrower is risk averse he consumes a constant positive quantity when the state is verified and he is in default (i.e. there is default insurance), but the verification regions, $S^{*}$ is still an interval of low return states. ${ }^{1}$

There is no reason to believe an optimizing entrepreneur is less risk averse than any other economic agent, and hence Gale and Hellwig's (1985) and Garino and Simmons'(20001) discussions of risk aversion reflect a desire to adapt the model to more realistic conditions. The assumption that the bank is risk neutral is realistic due to the bank's diversified loan portfolio, but this fact brings out a fundamental aspect of the contract. The bank's ability to diversify enables it to become an insurance agent for a risk averse borrower. The need for such insurance arises from the non-existence of markets for contingent claims which is due to asymmetric information. This means that private contracting between the borrower and the bank becomes an institution used to improve risk sharing arrangements that should ideally be performed via financial markets. But then the simple borrower-lender relationship no longer characterizes the problem; rather the relationship has become multifaceted. Nevertheless, under the assumptions of Gale and Hellwig(1985) and Garino and Simmons(2006), the formal structure of the standard loan contract remains intact.

Our interest in this paper is to extend the analysis of Gale and Hellwig(1985) to an environment where the borrower is risk averse but where he also has a probability belief about the distribution of the state $s$ which is different from that of the bank. In that case, in some states the agreed compensation may not be connected with the real outcome of the project but rather, it may be due to the differences in probabilities on which the parties trade. With this in mind, it may not be useful to think of $S^{*}$ as a set of default states. Instead, $S^{*}$ is the set of states in which the contract stipulates a payoff to the borrower that exceeds the project's resources

\footnotetext{
${ }^{1}$ If there are any non-verification states, then due to the bank's verification costs, there will typically be a discontinuity in the payoff schedule of the borrower at the point of transition from the verification to the non-verification interval. With a view to removing such discontinuity, and inspired by Innes'(1990) model of unobserved effort level, Garino and Simmons(2006) consider the idea that the borrower can destroy output. Under such an indirect assumption of endogenous effort, they show that the consumption level of the borrower is monotonic with no discontinuities in the state contingent schedule.
} 
minus the fixed obligation to the bank. The insuring agent then makes a demand for payment by the lender to cover his costs. More generally, the constraints do not prohibit the borrower from receiving a compensation which is even larger than the total resources of the entire project. In fact, for both the original Gale and Hellwig model and ours, the contract may be thought of as being composed of two contracts, one that in return for a loan from the the bank offers a state independent future payment to the bank and another which is an insurance contract with establishes state dependent pay-outs. Monitoring is then used to certify whether the insured event did or did not happen. These two contracts are necessarily linked because (under an assumption of non-negative consumption) the promises of the pure loan contract are not feasible without the insurance contract, thus the loan contract will stipulate that an insurance contract be signed. The linkage is only one-way however and with subjective expectations, under some circumstances, an insurance contract may be signed even when there is no lending by the bank. Seen in this light a natural question is then, whether the two contracts necessarily have to be offered by the same financial institution? We argue informally that consolidating the two contracts into one has the advantage to the bank of guaranteeing that the second contract (in its stipulated format) is in fact in place and of making the risk profile of the insured known. If there are fixed costs associated with writing up a contract this may be a further reason why a single contract is preferable.

To explain the issue further, consider the case when $S^{*}$ is an interval of lowest returns. Due to risk aversion (and possibly, probability differences) the lender proposes to insure the borrower in terms of a specified payoff function on $S^{*}$. In addition, suppose there is another region $S^{* *}$, not connected with $S^{*}$, on whose realization the lender puts very small probability but the borrower very high probability. The borrower may ask to receive a large compensation on $S^{* *}$ and, in addition, that on $S^{* *}$ the project should have no obligation to the bank. The lending bank may be willing to guarantee the borrower this compensation package simply because he does not believe that these outcomes are likely. If the lender can lower the amounts proposed to the borrower at $S^{*}$ (a region of high probability for him) in exchange for higher promises on the set $S^{* *}$ (a region of low probability for him), such a trade would keep the expected return of the lender at the same level and increase the utility of the borrower. One may then argue that trading on probability differences introduces an element of "speculation" something which is not the proper function of a lending bank. But this is exactly the point at which the bank's motive to maximize expected return alters its simple role as a loan provider and turns it into a trading partner in an environment of incomplete markets.

It is worth noting that in recent years many economists have adopted the view that incomplete markets should not be characterized only by an absence of financial markets to trade risk but also by the emergence of private institutions to supplement trading opportunities available 
in the market. Indeed, some theorists consider contracting to be the correct way to model incomplete markets (e.g. Atkeson and Lucas, 1992, Cole and Kocherlakota, 2001). Without addressing this deeper question of selecting optimal private institutions to trade risk, we note that a crucial feature of contracting with risk aversion and diverse probability beliefs is the fact that it offers a substitute for financial markets, and the possibility of multiple regions of verification simply reflects the complex nature of trading it implies. As was already noted, in the environment we consider the financial contract is a bundle who's elements cannot easily be separated. ${ }^{2}$

Bernanke, Gertler and Gilchrist(1999) use the contracting model to develop a financial accelerator and in applying the model to the U.S. economy take an extreme position on this issue. They argue that in advanced economies there always exists intermediation between the ownership of capital and the management of capital, hence all public capital ownership is covered by the state verification model! They thus do not distinguish between equity and debt investments since they are both subject to state verification. However, the Gale and Hellwig(1985) assumptions, adopted by Bernanke, Gertler and Gilchrist(1999), seem to lead to a too simple contract rather than the very complex set of contingent obligations and payments, typical associated with capital investment. Despite this shortcoming, the approach by Bernanke and Gertler (1989) Bernanke, Gertler and Gilchrist(1999), and Carlstrom and Fuerst(1997) has become influential in the macroeconomic literature since it offers a relatively simple way of introducing into a new Keynesian model a role for the financial markets and in particular the possibility that these markets are accelerating, i.e. magnifying, real shocks to the economy. Our intuition is that when entrepreneurs are optimistic, as we assume here, the accelerator affect will tend to become more pronounced, since the entrepreneurs tend to take on more risk than they otherwise would.

Our interest in contrasting with the established literature naturally lead us to adopt one common assumption there, namely that monitoring is non-stochastic. However, both Townsend (1979) and, for a more general case, Mookherjee and Png(1989) showed that the optimal contract in general involves stochastic monitoring. This means that a contractual format quite different from the standard loan contract of Gale and Hellwig(1985) emerges. Conceptually it is not straight forward to allow for stochastic monitoring in a setting with subjective beliefs like ours. In particular, the lender and borrower may disagree about the probabilities of default and they might see an advance in letting the monitoring depend on an event, which the

\footnotetext{
${ }^{2}$ Note that this way of thinking about the borrower-lender relationship may then also provide a perspective on the ongoing discussion about the merits of funding via equity markets as compared to via investment banks (and more recently investment funds). The latter may be attractive exactly because it simultaneous serves several needs of the firm.
} 
lender think has small probability and the borrower has high probability. The issue is further complicated by another assumption common to both the models of Gale and Helllwig(1985) and Mookherjee and Png(1989) namely that the lender can commit to monitor. In the first place if the decision to monitor is not triggered by a commonly observable event, it becomes more difficult to confirm that the commitment is fulfilled. Secondly, in the case of delegated monitoring there is when all parties have subjective beliefs a further source of complications in determining the optimal form of monitoring and controlling that it is implemented. In fact Mookherjee and Png(1989) themselves suggest that the format of the standard loan contract may be explained by simplicity and simplicity may also be the reason why deterministic monitoring is used. Finally, since a major motivation for extending the Gale-Hellwig model was to understand how subjective beliefs may affect the financial accelerator, we find it useful to retain the assumption of non-stochastic monitoring used in f.i. Bernanke, Gertler and Gilchrist (1999).

In generalizing the Gale and Hellwig model, there are many sound reasons to focus on the case where the lender is more optimistic than the bank. Firstly, there is vast empirical evidence that agents exhibit diverse probability beliefs in financial markets (see, for example, Frankel and Foot(1987),(1990), Frankel and Rose(1995), Kandel and Pearson(1995), Kurz(2001) and Takagi(1991)). Secondly, if potential entrepreneurs have diverse beliefs about available projects, we would expect that investors who are pessimistic about the outcomes of potential projects would generally choose not to invest at all. Thirdly, the case of differences in probability beliefs is a relatively simple setting of trading where we could hope to derive the optimal contract and even be able to solve it numerically. Other settings may be too complex to enable a full characterization of the optimal contract. And, indeed, our conclusions are relatively simple and can be summarized as follows:

(A) The contractual payments to the borrower in verification states are not constant, unless he and the bank agree on probability assessments: They vary by states in accord with risk aversion and probability beliefs of the borrower and lender.

(B) The observation region may consist of several intervals and the structure of these intervals is sensitive to the differences in probability beliefs.

The contract feature described in (A) has substantial empirical evidence to support it, since even in states of "default" the consumption level of a borrower is not constant. There are items (e.g. home) not subject to seizure by lender; in some states the borrower may default only on some loans which are not backed by collateral. Finally, depending on the severity of the situation (i.e. depending on $s$ ), if the borrower is unable to pay all his debts then under Chapter 11 he may be able to renegotiate the loan contracts and emerge with a positive consumption. 
The feature described in (B) reflects the trading possibilities which private contracting makes possible.

\section{Characterization of the Solution to the Contracting Problem}

Here the case where the payoff to a risk averse borrower is not required to be monotonic is being studied ${ }^{3}$. The following notation is used in our formulation:

$N$ - equity of the borrower;

$B$ - amount borrowed and capital $C=B+N$;

$S \subset \Re_{+}$- the state space;

$F: S \times \Re_{+} \rightarrow \Re_{+}$- the production function, a function of the state $s$ and the input of capital;

$P: S \rightarrow[0,1]$ - the distribution of $s$ according to the bank;

$P_{B}: S \rightarrow[0,1]$ - the distribution of $s$ according to the borrower;

$u: \Re_{+} \rightarrow \Re$ - the utility function of the borrower;

$c: S \times \Re_{+} \rightarrow \Re_{+}-$the verification cost depending on the state and the input of capital;

$S^{*} \subset S$ - the verification set: Set of states at which according to the contract observation takes place

$\nu: S \rightarrow \Re_{+}$- the state contingent payment to the borrower;

$R$ - payment to the bank (i.e. the loan $B$ plus contractual interest) when the state is unobserved ${ }^{4}$;

$r$ - riskless interest rate;

All functions and sets are required to be measurable. We start with five assumptions but add more later to facilitate the development.

Assumptions

A.1 $u$ is $C^{2}$ with $u^{\prime}>0, u^{\prime \prime}<0$;

A.2 The bank is risk neutral;

A.3 The probability measures $P$ and $P_{B}$ are equivalent;

\footnotetext{
${ }^{3}$ Garino and Simmons(2006) explore the implication of permitting the borrowing entrepreneur to destroy revenue and this results in an monotonic payoff function to the borrower.

${ }^{4}$ It is straightforward to see that in the region where there is no observation, since the bank relies on the report of the borrower, the payment to the bank must be constant, so that $\nu(s)=F(s, B+n)-R$ in this region.
} 
Without loss of generality we now let $S$ be the support of $P$ (or equivalently of $P_{B}$ ).

A.4 $\forall C: \int_{S} F(s, C) P(d s)<\infty, \int_{S} F(s, C) P_{B}(d s)<\infty$.

A.5 $S$ is connected and contains 0 ;

We are mainly interested in characterizing the optimal contract, whenever it exists, and could therefore at the present level of generality consider the case also of risk neutral borrowers. However, we consider this case as not of much interest, since even if an optimal contract exists, it will tend to be rather extreme, its format being driven by differences in opinions.

In the way we shall formulate the contract design problem we are implicitly assuming that beliefs are known. This may seem an extreme assumption, but less so when taking into account that there is, in the background of our analysis, an assumption of competitive markets. This is why we shall assume a zero profit participation constraint for the bank. We think of a situation with many competing banks, that may have different beliefs about the type of project offered by the entrepreneur. Each of them will accept any contract that according to their belief offers an expected return equal to zero. The entrepreneur will then choose the bank willing to accept the in his view most favorable contract. Assuming that there are many banks of each belief type, the zero profit condition must then hold in equilibrium. Note that the banks then have no interest in knowing the entrepreneur's belief (which might in any case be retrieved from the contract he proposes).

\subsection{A simple tool for characterizing the Solution of the Contracting Problem}

We start with a formal statement of the contracting problem which is:

Select $S^{*}$ measurable, $\nu(\cdot) \geq 0$ defined on $S$ and measurable, $R \geq 0, B \geq 0$ that, with $\nu(s)=F(s, B+N)-R$ on $S \backslash S^{*}$, maximize:

$$
\begin{array}{r}
\int_{S^{*}} u[\nu(s)] P_{B}(d s)+\int_{S \backslash S^{*}} u[F(s, B+N)-R] P_{B}(d s) \\
\text { subject to } \\
\int_{S^{*}}[F(s, B+N)-\nu(s)-c(s, B+N)] P(d s)+\int_{S \backslash S^{*}} R P(d s) \geq B(1+r) \\
\nu(s) \geq F(s, N+B)-R, \forall s \in S^{*} \\
F(s, N+B)-R \geq 0, \forall s \in S \backslash S^{*}
\end{array}
$$

In this problem a feasible contract is one that pays a non-negative amount to the entrepreneur in any state (which could be interpreted as limited liability). The first constraint, ( 2 ) describes the participation constraint of the bank, ensuring the bank a normal return, (3) is the 
incentive compatibility constraint making it unprofitable for the borrower to misrepresent the truth by claiming that a state in $S^{*}$ belongs to $S \backslash S^{*}$, while (4) is a feasibility constraint on the contractual value of $R .^{5}$ We denote by $\tilde{\nu}, \tilde{S}^{*}, \tilde{R}, \tilde{B}$ a solution to this problem (which we throughout shall assume exists, unless otherwise specified). Note the in many instances the solution is not unique but is a class of probabilistic equivalent solutions.

\section{Remark 1}

If $c(s, \tilde{B}+N)>0, \forall s, \forall C,(3)$ will almost surely not bind. For if $\tilde{\nu}=F(s, \tilde{B}+N)-\tilde{R}$ on a set $G$ of positive measure, excluding $G$ from the set of observations while leaving the payoff to the borrower unchanged is profitable and would, because $c(s, \tilde{B}+N)>0$ on $G$, be an improvement

Our strategy for characterizing a solution to (1) - (4) is to define a simpler problem who's solution is part of the solution to the original problem. Thus, for given $C$ and $k \in \Re_{+}$, denote by $\hat{\nu}_{C}(\cdot, k)$ the solution to the following problem (5) - (6) (whenever it exists):

Select $\nu(\cdot) \geq 0$, measurable which maximizes

$$
\begin{array}{r}
\int_{S} u[\nu(s)] P_{B}(d s) \\
\text { subject to } \\
\int_{S}[F(s, C)-\nu(s)] P(d s) \geq k
\end{array}
$$

Problem (5) - (6) is much simpler than problem (1) - (4) and we shall find first order conditions for it. Note that for any measurable $\tilde{S}, \hat{\nu}_{C}(\cdot, k)$ restricted to $\tilde{S}$ is the solution to $\max \int_{\tilde{S}} u[\nu(s)] P_{B}(d s)$ s.t. to $\int_{\tilde{S}}[F(s, C)-\nu(s)] P(d s) \geq k-\int_{S \backslash \tilde{S}}[F(s, C)-\nu(s)] P(d s)$.

Let $C$ and $k$ be given such that (5) - (6) has a solution (i.e. $\int_{S} F(s, C) \geq k$ ). With $S_{0 \delta}=$ $\left\{s \in S: \hat{\nu}_{C}(s, k)>\delta\right\}$ we then have the following necessary condition for optimality: For any measurable $A, B \subset S$ and $\epsilon>0$ such that $P(B)>0$ and $P(B)=P\left[B \cap S_{0 \epsilon \frac{P(A)}{P(B)}}\right]$

$$
\int_{A}\left[u\left(\hat{\nu}_{C}(s, k)+\epsilon\right)-u\left(\hat{\nu}_{C}(s, k)\right)\right] P_{B}(d s)+\int_{B}\left[u\left(\hat{\nu}_{C}(s, k)-\epsilon \frac{P(A)}{P(B)}\right)-u\left(\hat{\nu}_{C}(s, k)\right)\right] P_{B}(d s) \leq 0
$$

Dividing by $\epsilon$ and taking limits we conclude that in the case where $P\left(B \cap S_{00}\right)=P(B)>0$ :

$$
\int_{A} u^{\prime}\left[\hat{\nu}_{C}(s, k)\right] P_{B}(d s)-\int_{B} u^{\prime}\left[\hat{\nu}_{C}(s, k)\right] \frac{P(A)}{P(B)} P_{B}(d s) \leq 0
$$

\footnotetext{
${ }^{5}$ Note that we implicitly assume a penalty for falsely claiming a state in $S^{*}$ (which will always be revealed) sufficiently high to deter such misreporting.
} 
Likewise, if $P(B)>0$ and $P\left(A \cap S_{00}\right)=P(A)$,

$$
\int_{A} u^{\prime}\left[\hat{\nu}_{C}(s, k)\right] P_{B}(d s)-\int_{B} u^{\prime}\left[\hat{\nu}_{C}(s, k)\right] \frac{P(A)}{P(B)} P_{B}(d s) \geq 0
$$

The key tools to characterizing the solution to the contracting problem are Lemmas 1 and 2 , both of which involve standard arguments. They show the format of the payoff function in the optimal verification set, $\tilde{S}^{*}$.

\section{Lemma 1}

Suppose $k_{n} \downarrow k$ with $\hat{\nu}_{C}(s, k)$ and $\hat{\nu}_{C}\left(s, k_{n}\right)$ well defined. Then $\hat{\nu}_{C}\left(s, k_{n}\right) \uparrow \hat{\nu}_{C}(s, k)$ for $P$-a.a. $s$.

Proof: We first prove monotonicity by use of the first order conditions, (8) and (9). Thus suppose $k>k^{\prime}$ and let $\bar{A}=\left\{s \in S: \hat{\nu}_{C}(s, k)>\hat{\nu}_{C}\left(s, k^{\prime}\right)\right\}$ and $\tilde{B}=\left\{s \in S: \hat{\nu}_{C}(s, k)<\hat{\nu}_{C}\left(s, k^{\prime}\right)\right\}$. We have $P(\tilde{B})>0$. Suppose $P(\bar{A})>0$ with the purpose of arriving at a contradiction. Using (8) we have

$$
\frac{\int_{\bar{A}} u^{\prime}\left[\hat{\nu}_{C}\left(s, k^{\prime}\right)\right] P_{B}(d s)}{\int_{\tilde{B}} u^{\prime}\left[\hat{\nu}_{C}\left(s, k^{\prime}\right)\right] P_{B}(d s)} \leq \frac{P(\bar{A})}{P(\tilde{B})}
$$

Next using (9) we have:

$$
\frac{P(\bar{A})}{P(\tilde{B})} \leq \frac{\int_{\bar{A}} u^{\prime}\left[\hat{\nu}_{C}(s, k)\right] P_{B}(d s)}{\int_{\tilde{B}} u^{\prime}\left[\hat{\nu}_{C}(s, k)\right] P_{B}(d s)}
$$

so that

$$
\frac{\int_{\bar{A}} u^{\prime}\left[\hat{\nu}_{C}\left(s, k^{\prime}\right)\right] P_{B}(d s)}{\int_{\tilde{B}} u^{\prime}\left[\hat{\nu}_{C}\left(s, k^{\prime}\right)\right] P_{B}(d s)} \leq \frac{P(\bar{A})}{P(\tilde{B})} \leq \frac{\int_{\bar{A}} u^{\prime}\left[\hat{\nu}_{C}(s, k)\right] P_{B}(d s)}{\int_{\tilde{B}} u^{\prime}\left[\hat{\nu}_{C}(s, k)\right] P_{B}(d s)}
$$

which is impossible, since on $\bar{A}, u^{\prime}\left[\hat{\nu}_{C}\left(s, k^{\prime}\right)\right]>u^{\prime}\left[\hat{\nu}_{C}(s, k)\right]$ while on $\tilde{B}$, we have $u^{\prime}\left[\hat{\nu}_{C}\left(s, k^{\prime}\right)\right]<$ $u^{\prime}\left[\hat{\nu}_{C}(s, k)\right]$. We thus conclude that $P(\bar{A})=0$. This proves almost sure monotonicity.

Now let $k_{n} \downarrow k$. Then there is a measurable $\bar{\nu}$ s.t. for $P$-a.a. $s, \hat{\nu}_{C}\left(s, k_{n}\right) \uparrow \bar{\nu}(s)$. Suppose that on some measurable $\bar{B}$ with $P(\bar{B})>0, \bar{\nu}<\hat{\nu}_{C}(\cdot, k)$. Since

$$
\int_{S}[F(s, C)-\bar{\nu}(s)] P(d s)=k=\int_{S}\left[F(s, k)-\hat{\nu}_{C}(s, k)\right] P(d s)
$$

there must be some measurable set $B^{\prime}$ with $P\left(B^{\prime}\right)>0$ s.t. on $B^{\prime}, \bar{\nu}>\hat{\nu}_{C}(\cdot, k)$. But this is in contradiction with the almost sure monotonicity that was just established, since then there would be a measurable subset of $B^{\prime}$ with $P$-positive measure on which for large $n, \hat{\nu}_{C}\left(\cdot, k_{n}\right)>$ $\hat{\nu}(\cdot, k)$. Hence $\bar{\nu}=\hat{\nu}_{C}(\cdot, k)$ a.e. and convergence has been proven

Note that if $u^{\prime}(0)=\infty, \hat{\nu}_{C}(s, k)>0, \forall s$ and the lemma still applies. 


\section{Lemma 2}

Let $\tilde{\nu}, \tilde{S}^{*}, \tilde{R}$, and $\tilde{B}$ be a solution to the contracting problem $(1)$ - (4). Assume $c(s, C)>0, \forall s, C$. Then there is a $\tilde{k}$ s.t. $\tilde{\nu}=\hat{\nu}_{\tilde{C}}(s, \tilde{k})$ for $P$-a.a. $s \in \tilde{S}^{*}$ with $\tilde{C}=N+\tilde{B}$.

Proof: In the following we consider the case where $P\left(S^{*}\right)>0$, else the lemma trivially holds. Let $\hat{k}=\tilde{B}(1+r)-P\left(S \backslash \tilde{S}^{*}\right) \tilde{R}+\int_{\tilde{S}^{*}} c(s, \tilde{B}+N) P(d s)$. Then $\tilde{\nu}$ restricted to $\tilde{S}^{*}$ solves the problem:

Select $\nu(\cdot) \geq 0$, measurable and defined on $\tilde{S}^{*}$ which maximizes:

$$
\begin{array}{r}
\int_{\tilde{S}^{*}} u[\nu(s)] P_{B}(d s) \\
\text { subject to } \\
\int_{\tilde{S}^{*}}[F(s, \tilde{B}+N)-\nu(s)] P(d s) \geq \hat{k} \\
\nu(s) \geq F(s, \tilde{B}+N)-\tilde{R}, \forall s \in \tilde{S}^{*}
\end{array}
$$

Remark 1 tells us that (12) is non-binding for $\tilde{\nu}$. Let $\hat{\nu}$ solve $\max (10)$ subject to (11). If $\hat{\nu} \neq \tilde{\nu}$ on a set in $\tilde{S}^{*}$ of positive measure then

$$
\int_{\tilde{S}^{*}} u[\hat{\nu}(s)] P_{B}(d s)>\int_{\tilde{S}^{*}} u[\tilde{\nu}(s)] P_{B}(d s)
$$

This follows from strict concavity of $u$. Suppose then, with the purpose of obtaining a contradiction, that with $\hat{S}=\left\{s \in \tilde{S}^{*}: \hat{\nu}(s) \neq \tilde{\nu}(s)\right\}, P(\hat{S})>0$. Let

$$
\hat{S}_{L}=\{s \in \hat{S}: F(s, \tilde{C})-\tilde{R} \geq L\}
$$

and, by A.4, $P\left(\hat{S}_{L}\right) \rightarrow 0$ as $L \rightarrow \infty$. Define, for large $L, \hat{\nu}_{L}$ on $\tilde{S}^{*}$ by:

$$
\begin{array}{r}
\hat{\nu}_{L}(s)=\tilde{\nu}(s) \text { for } s \in \tilde{S}^{*} \backslash\left(\hat{S} \backslash \hat{S}_{L}\right) \\
\hat{\nu}_{L}(s)=\max \left\{0, \hat{\nu}(s)-\delta_{L}\right\} \text { for } s \in \hat{S} \backslash \hat{S}_{L}
\end{array}
$$

where $\delta_{L}$ is chosen s.t.

$$
\int_{\hat{S}} \hat{\nu}_{L} d P=\int_{\hat{S}} \hat{\nu} d P
$$

As $L \rightarrow \infty, \hat{\nu}_{L}(s) \rightarrow \hat{\nu}(s)$ a.s., so there is an $\bar{L}$, s.t. $\int_{\tilde{S}^{*}} u\left[\hat{\nu}_{\bar{L}}(s)\right] P_{B}(d s)>\int_{\tilde{S}^{*}} u[\tilde{\nu}(s)] P_{B}(d s)$ and $P\left\{s \in \hat{S} \backslash \hat{S}_{\bar{L}}: \hat{\nu}_{\bar{L}}(s)>0\right\}>0$. Let $S^{\prime}=\hat{S} \backslash \hat{S}_{\bar{L}}$.

To any $\epsilon>0$ assign an $\eta_{\epsilon}>0$ s.t. with $S_{\epsilon}=\left\{s \in S^{\prime}: \tilde{\nu}(s)-F(s, \tilde{C})-\tilde{R}<\eta_{\epsilon}\right\}, P\left(S_{\epsilon}\right)<\epsilon$. For small $\epsilon>0$, modify $\hat{\nu}_{\bar{L}}$ as follows:

$$
\begin{aligned}
& \hat{\nu}_{\epsilon}(s)=\max \left\{F(s, \tilde{B}+N)-\tilde{R}, \hat{\nu}_{\bar{L}}(s)\right\}, \text { if } s \in S_{\epsilon} \\
& \hat{\nu}_{\epsilon}(s)=\max \left\{0, \hat{\nu}_{\bar{L}}(s)-\delta\right\} \text { if } s \in S^{\prime} \backslash S_{\epsilon} \\
& \quad \text { where } \delta \geq 0 \text { is chosen such that } \int_{S^{\prime}} \hat{\nu}_{\epsilon}(s) P(d s)=\int_{S^{\prime}} \hat{\nu}_{\bar{L}}(s) P(d s)
\end{aligned}
$$

$\hat{\nu}_{\epsilon}(s)=\hat{\nu}_{\bar{L}}(s)$, else 
Note that $S_{\epsilon}$ may be empty in which case $\delta=0$ and $\hat{\nu}_{\epsilon}=\hat{\nu}_{\bar{L}}$. Now, as $\epsilon \downarrow 0, \hat{\nu}_{\epsilon} \rightarrow \hat{\nu}_{\bar{L}}$ a.s. so there is an $\bar{\epsilon}>0$ for which $\int_{\tilde{S}^{*}} u\left[\hat{\nu}_{\bar{\epsilon}}(s)\right] P_{B}(d s)>\int_{\tilde{S}^{*}} u[\tilde{\nu}(s)] P_{B}(d s)$. For such an $\bar{\epsilon}$, pick $\lambda \in(0,1)$ such that

$$
\lambda \hat{\nu}_{\bar{\epsilon}}(s)+(1-\lambda) \tilde{\nu}(s) \geq F(s, \tilde{B}+N)-\tilde{R}, \forall s \in S^{\prime}
$$

This is possible because on $S_{\bar{\epsilon}}, \hat{\nu}_{\bar{\epsilon}}(s) \geq F(s, \tilde{B}+N)-\tilde{R}$ and $\tilde{\nu}(s)>F(s, \tilde{B}+N)-\tilde{R}$ (by Remark 1), while on $S^{\prime} \backslash S_{\bar{\epsilon}}, F(\cdot, \tilde{C})$ is bounded by $\bar{L}, \tilde{\nu}(s) \geq F(s, \tilde{B}+N)-\tilde{R}+\eta$ for some $\eta>0$ and $\hat{\nu}_{\bar{\epsilon}} \geq 0$. By strict concavity of $u$ we have

$$
u\left[\lambda \hat{\nu}_{\bar{\epsilon}}(s)+(1-\lambda) \tilde{\nu}(s)\right] \geq \lambda u\left[\hat{\nu}_{\bar{\epsilon}}(s)\right]+(1-\lambda) u[\tilde{\nu}(s)], \forall s \in S^{\prime}
$$

with strict inequality in a set of positive $P$-measure. Hence we have

$$
\int_{\tilde{S}^{*}} u\left[\lambda \hat{\nu}_{\bar{\epsilon}}(s)+(1-\lambda) \tilde{\nu}(s)\right] P_{B}(d s)>\lambda \int_{\tilde{S}^{*}} u\left[\hat{\nu}_{\bar{\epsilon}}(s)\right] P_{B}(d s)+(1-\lambda) \int_{\tilde{S}^{*}} u[\tilde{\nu}(s)] P_{B}(d s)
$$

which implies that

$$
\int_{\tilde{S}^{*}} u\left[\lambda \hat{\nu}_{\bar{\epsilon}}(s)+(1-\lambda) \tilde{\nu}(s)\right] P_{B}(d s)>\int_{\tilde{S}^{*}} u[\tilde{\nu}(s)] P_{B}(d s) .
$$

However, by construction $\lambda \hat{\nu}_{\bar{\epsilon}}+(1-\lambda) \tilde{\nu}$ fulfills the two constraints (11) and (12). This contradicts the fact that $\tilde{\nu}$ was a solution to $(10)-(12)$ and it must be the case that $\tilde{\nu}=\hat{\nu}$ on $\tilde{S}^{*}$. What remains to be shown is that for some $\tilde{k}$

$$
\hat{\nu}(s)=\hat{\nu}_{\tilde{C}}(s, \tilde{k}), \forall s \in \tilde{S}^{*}
$$

Clearly, as $k \uparrow \int_{0}^{\infty} F(s, \tilde{C}) P(d s), \hat{\nu}_{\tilde{C}}(\cdot, k) \downarrow 0, P$-a.s. which means $\int_{\tilde{S}^{*}} \hat{\nu}_{\tilde{C}}(s, k) P_{B}(d s) \downarrow 0$. On the other hand, as $k \downarrow-\infty, \hat{\nu}_{\tilde{C}}(\cdot, k) \uparrow \infty, P$-a.s. by the same argument used earlier to prove monotonicity in Lemma 1 . Lemma 1 establishes the pointwise continuity of $\hat{\nu}_{\tilde{C}}$, hence the continuity (in $k$ ) of $\int_{\tilde{S}^{*}} \hat{\nu}_{C}(s, k) P(d s)$ so (by the intermediate value theorem) it follows that there is a $\tilde{k}$ such that

$$
\int_{\tilde{S}^{*}} \hat{\nu}_{\tilde{C}}(s, \tilde{k}) P(d s)=\int_{\tilde{S}^{*}} F(s, \tilde{C}) P(d s)-\hat{k}
$$

Since $\hat{\nu}_{\tilde{C}}(\cdot, \tilde{k})$ is the unique solution to the problem $\max \int_{\tilde{S}^{*}} u[\nu(s)] P_{B}(d s)$ subject to $\int_{\tilde{S}^{*}} \nu(s) P(d s)$ $\geq \int_{\tilde{S}^{*}} \hat{\nu}_{\tilde{C}}(s, \tilde{k}) P(d s),(13)$ follows

Lemma 2 has a simple interpretation. Since in $\tilde{S}^{*}$ the state is known, the problem restricted to $\tilde{S}^{*}$ is an optimization with full information. Hence the borrower's consumption must be optimal only subject to the participation constraint of the bank i.e. to the condition that $\tilde{k}$ is the level that assures the bank's participation. Given that this conditions is satisfied, the optimality condition (8) simply reflects the risk aversion and probability belief of the borrower as well as the probability belief of the bank. We recall that for the case where the borrower 
is risk averse but holds the same probability belief as the bank, Gale and Hellwig(1985) and Garino and Simmons(2006) show the solution is $\tilde{\nu}=$ constant for $s \in \tilde{S}^{*}$. If the agent is risk neutral and holds the same probability belief as the bank then we have the standard loan contract where $\tilde{\nu}(s)=0, s \in \tilde{S}^{*}$. The new dimension here is the diversity of probability beliefs between the bank and the borrower.

Lemma 2 enables us to show that when sufficient smoothness is assumed, the solution to (1) - (4) can be characterized with elementary techniques of calculus.

\subsection{Necessary Conditions for the optimal solution $\tilde{\nu}$}

We have already derived in (8) and (9) necessary conditions for problem (5) - (6). We now combine with the previous lemma to derive necessary conditions for problem (1) - (4).

Necessary Conditions for an Optimal Pay-off Function $\tilde{\nu}$

For all measurable $A, B \subset \tilde{S}^{*}$ s.t. $P(B)>0$ and $\tilde{\nu}(s)>0$ for $P$-a.a. $s \in A \cup B$ it is necessary that

$$
\int_{A} u^{\prime}[\tilde{\nu}(s)] P_{B}(d s)-\int_{B} u^{\prime}[\tilde{\nu}(s)] \frac{P(A)}{P(B)} P_{B}(d s)=0
$$

If $P$ and $P_{B}$ are differentiable in $s$ and $s^{\prime}$, so that they have densities $p$ and $p_{B}$ defined in $s$ and $s^{\prime},(14)$ can be rewritten in the form

$$
\frac{u^{\prime}[\tilde{\nu}(s)] p_{B}(s)}{u^{\prime}\left[\tilde{\nu}\left(s^{\prime}\right)\right] p_{B}\left(s^{\prime}\right)}=\frac{p(s)}{p\left(s^{\prime}\right)} \text { if } \tilde{\nu}(s), \tilde{\nu}\left(s^{\prime}\right)>0, p\left(s^{\prime}\right)>0, p_{B}\left(s^{\prime}\right)>0
$$

Note that when $P_{B}=P,(14)$ implies that $\tilde{\nu}$ is a.e. constant on $\tilde{S}^{*}$ as in Gale and Hellwig(1985). Note also that when $p_{B}$ and $p$ are continuous, so is, a.e., $\tilde{\nu}$ restricted to $\tilde{S}^{*}$.

Given the choice of capital, $\tilde{C}=\tilde{B}+N$, a contract specifies $\tilde{S}^{*}, \tilde{k}$ and $\tilde{R}$ with $\tilde{\nu}(s)=\hat{\nu}_{\tilde{C}}(s, \tilde{k})$ for $s \in \tilde{S}^{*}$ and $\tilde{\nu}(s)=F(s, \tilde{C})-\tilde{R}$ for $s \in S \subset \tilde{S}$. Hence, we can develop necessary conditions for optimality in terms of the three choice variables, $S^{*}, k$, and $R$. First, we make additional assumptions:

A.6 $F$ is continuous and weakly increasing in $s$.

A.7 $c$ is continuous in $s$ and $c(\cdot, C)>0, \forall s \in S, \forall C>0$.

A.8 $\quad P_{B}$ and $P$ have densities, $p_{B}$ and $p$ which are continuous.

Notice that we can always reorder S, in order to obtain A.6.

The assumption that $c(\cdot, C)>0$ is made for simplicity and our results could be derived with the less strict condition that $\{s \in S: c(s, C)=0\}$ be independent of $C$. In that case we would have to restrict the analysis to the set where $c(s, C)>0, \forall C$, knowing that on the complement set, there will always be observation. 
For a given optimal contract, $\left(\tilde{C}, \tilde{k}, \tilde{S}^{*}, \tilde{R}\right)$, let $S_{0}=\{s \in S: F(s, \tilde{C})-\tilde{R}<0\}$ so $S_{0} \subset \tilde{S}^{*}$. Also observe that

$$
S_{1} \equiv\left\{s \in S: \hat{\nu}_{\tilde{C}}(s, \tilde{k}) \leq F(s, \tilde{C})-\tilde{R}\right\} \subset S \backslash \tilde{S}^{*},
$$

a conclusion which follows from incentive compatibility. In the following we want to develop conditions to characterize what members of the set

$$
\bar{S} \equiv\left\{s \in S: \hat{\nu}_{\tilde{C}}(s, \tilde{k})>F(s, \tilde{C})-\tilde{R} \geq 0\right\}
$$

belong to $\tilde{S}^{*}$ and $S \backslash \tilde{S}^{*}$ respectively.

\section{Proposition 1}

Suppose, for an optimal contract $\left(\tilde{B}, \tilde{S}^{*}, \tilde{k}, \tilde{R}\right)$ with $\tilde{C}=\tilde{B}+N$, that $P\left(\tilde{S}^{*}\right)>0$ and $P\left(S \backslash \tilde{S}^{*}\right)>$ 0 . For any compact interval $I$, s.t. $\hat{\nu}_{\tilde{C}}(\cdot, \tilde{k})>0$ on $I$ and, with $\hat{S}^{*}=I \cap \tilde{S}^{*}, P\left(\hat{S}^{*}\right)>0$, we have for $P$-a.a. $s^{\prime} \in \tilde{S}^{*} \cap \bar{S}$ :

$$
\frac{u\left[\hat{\nu}_{\tilde{C}}\left(s^{\prime}, \tilde{k}\right)\right]-u\left[F\left(s^{\prime}, \tilde{C}\right)-\tilde{R}\right]}{\hat{\nu}_{\tilde{C}}\left(s^{\prime}, \tilde{k}\right)-\left[F\left(s^{\prime}, \tilde{k}\right)-\tilde{R}\right]+c\left(s^{\prime}, \tilde{C}\right)} \frac{p_{B}\left(s^{\prime}\right)}{p\left(s^{\prime}\right)}-\frac{\int_{\hat{S}^{*}} u^{\prime}\left[\hat{\nu}_{\tilde{C}}(s, \tilde{k})\right] P_{B}(d s)}{P\left[\hat{S}^{*}\right]} \geq 0
$$

- and for $P$-a.a. $s^{\prime} \in\left(S \backslash \tilde{S}^{*}\right) \cap \bar{S}$ :

$$
\frac{u\left[\hat{\nu}_{\tilde{C}}\left(s^{\prime}, \tilde{k}\right)\right]-u\left[F\left(s^{\prime}, \tilde{C}\right)-\tilde{R}\right]}{\hat{\nu}_{\tilde{C}}\left(s^{\prime}, \tilde{k}\right)-\left[F\left(s^{\prime}, \tilde{k}\right)-\tilde{R}\right]+c\left(s^{\prime}, \tilde{C}\right)} \frac{p_{B}\left(s^{\prime}\right)}{p\left(s^{\prime}\right)}-\frac{\int_{\hat{S}^{*}} u^{\prime}\left[\hat{\nu}_{\tilde{C}}(s, \tilde{k})\right] P_{B}(d s)}{P\left[\hat{S}^{*}\right]} \leq 0
$$

Proof: We consider throughout the proof a continuous version of $\hat{\nu}_{\tilde{C}}(\cdot, \tilde{k})$. We first prove $(16)$ by contradiction. Thus suppose that $s^{\prime} \in \operatorname{int} \bar{S} \cap \tilde{S}^{*}$ and that $P\left(\tilde{S}^{*} \cap\left[s^{\prime}-\epsilon, s^{\prime}+\epsilon\right]\right)>0, \forall \epsilon>0$ (which holds $P$-a.s. on int $\bar{S} \cap \tilde{S}^{*}$ ). If $<$ in (16) there is an interval $\bar{I}=\left[s^{\prime}-\bar{\epsilon}, s+\bar{\epsilon}\right]$ (with $\bar{\epsilon}>0)$ s.t. $\forall s, \hat{s} \in \bar{I}$ :

$$
\frac{\left\{u\left[\hat{\nu}_{\tilde{C}}(s, \tilde{k})\right]-u[F(s, \tilde{k})-\tilde{R}]\right\}}{\left\{\hat{\nu}_{\tilde{C}}(\hat{s}, \tilde{k})-[F(\hat{s}, \tilde{k})-\tilde{R}]+c(\hat{s}, \tilde{C})\right\}} \frac{p_{B}(s)}{p(\hat{s})}-\frac{\int_{\hat{S}^{*}} u^{\prime}[\hat{\nu}(s, \tilde{k})] P_{B}(d s)}{P\left[\hat{S}^{*}\right]}<0
$$

Let

$$
K=\max _{s \in \bar{I}}\left\{u\left[\hat{\nu}_{\tilde{C}}(s, \tilde{k})\right]-u[F(s, \tilde{k})-\tilde{R}]\right\} p_{B}(s)
$$

and

$$
H=\min _{s \in \bar{I}}\left\{\hat{\nu}_{\tilde{C}}(s, \tilde{k})-[F(s, \tilde{C})-\tilde{R}]+c(s, \tilde{C})\right\} p(s)
$$

and, for sufficiently small $\epsilon \in(0, \bar{\epsilon})$, define implicitly $\delta(\epsilon)$ and $\hat{\delta}(\epsilon)$ as follows:

$$
\int_{\tilde{S}^{*} \cap\left[s^{\prime}-\epsilon, s^{\prime}+\epsilon\right]} H d s-\int_{\hat{S}^{*} \backslash\left[s^{\prime}-\epsilon, s^{\prime}+\epsilon\right]} \delta(\epsilon) p(s) d s=0
$$




$$
-\int_{\tilde{S}^{*} \cap\left[s^{\prime}-\epsilon, s^{\prime}+\epsilon\right]} K d s+\int_{\hat{S}^{*} \backslash\left[s^{\prime}-\epsilon, s^{\prime}+\epsilon\right]}\left\{u\left[\hat{\nu}_{\tilde{C}}(s, \tilde{k})+\hat{\delta}(\epsilon)\right]-u\left[\hat{\nu}_{\tilde{C}}(s, \tilde{k})\right]\right\} p_{B}(s) d s=0
$$

We then consider the following modification of the contract: Change the verification set $\tilde{S}^{*}$ to $\tilde{S}^{*} \backslash\left[s^{\prime}-\epsilon, s^{\prime}+\epsilon\right]$ and on $\hat{S}^{*} \backslash\left[s^{\prime}-\epsilon, s^{\prime}+\epsilon\right]$ change the payment to the entrepreneur to $\hat{\nu}_{\tilde{C}}(s, \tilde{k})+\hat{\delta}(\epsilon)$ and reduce the payment to the bank by $\delta(\epsilon)$.

The change in the profit of the bank is for $\epsilon \in(0, \bar{\epsilon})$ and small:

$$
\int_{\tilde{S}^{*} \cap\left[s^{\prime}-\epsilon, s^{\prime}+\epsilon\right]}\left\{\hat{\nu}_{\tilde{C}}(s, \tilde{k})+c(s, \tilde{C})-[F(s, \tilde{C})-\tilde{R}]\right\} p(s) d s-\int_{\hat{S}^{*} \backslash\left[s^{\prime}-\epsilon, s^{\prime}+\epsilon\right]} \delta(\epsilon) p(s) d s \geq 0
$$

while the change in utility of the entrepreneur is:

$$
\begin{array}{r}
-\int_{\tilde{S}^{*} \cap\left[s^{\prime}-\epsilon, s^{\prime}+\epsilon\right]}\left\{u\left[\hat{\nu}_{\tilde{C}}(s, \tilde{k})\right]-u[F(s, \tilde{k})-\tilde{R}]\right\} p_{B}(s) d s+ \\
\int_{\hat{S}^{*} \backslash\left[s^{\prime}-\epsilon, s^{\prime}+\epsilon\right]}\left\{u\left[\hat{\nu}_{\tilde{C}}(s, \tilde{k})+\hat{\delta}(\epsilon)\right]-u\left[\hat{\nu}_{\tilde{C}}(s, \tilde{k})\right]\right\} p_{B}(s) d s \geq 0
\end{array}
$$

To get the desired contradiction we need to show that there is $\bar{\epsilon}>\epsilon>0$ s.t. $\hat{\delta}(\epsilon)<\delta(\epsilon)$ i.e. such that not only is the changes feasible but we can, by adding some to the payment to the entrepreneur, make him strictly better off.

Letting $\lambda$ be Lebesgue measure, we have

$$
\delta(\epsilon)=\frac{H \lambda\left[\tilde{S}^{*} \cap\left[s^{\prime}-\epsilon, s^{\prime}+\epsilon\right]\right]}{P\left[\hat{S}^{*} \backslash\left[s^{\prime}-\epsilon, s^{\prime}+\epsilon\right]\right]}
$$

Using the identity

$$
\begin{gathered}
\hat{\delta}(\epsilon)=\frac{K \lambda\left[\tilde{S}^{*} \cap\left[s^{\prime}-\epsilon, s^{\prime}+\epsilon\right]\right]}{\int_{\hat{S}^{*} \backslash\left[s^{\prime}-\epsilon, s^{\prime}+\epsilon\right]} u^{\prime}\left[\hat{\nu}_{\tilde{C}}(s, \tilde{k})\right] p_{B}(s) d s}-\frac{\hat{\delta}(\epsilon)}{\int_{\hat{S}^{*} \backslash\left[s^{\prime}-\epsilon, s^{\prime}+\epsilon\right]} u^{\prime}\left[\hat{\nu}_{\tilde{C}}(s, \tilde{k})\right] p_{B}(s) d s} \\
\left\{\frac{\int_{\hat{S}^{*} \backslash\left[s^{\prime}-\epsilon, s^{\prime}+\epsilon\right]}\left\{u[\hat{\nu} \tilde{C}(s, \tilde{k})+\hat{\delta}(\epsilon)]-u\left[\hat{\nu}_{\tilde{C}}(s, \tilde{k})\right]\right\} p_{B}(s) d s}{\hat{\delta}(\epsilon)}-\int_{\hat{S}^{*} \backslash\left[s^{\prime}-\epsilon, s^{\prime}+\epsilon\right]} u^{\prime}\left[\hat{\nu}_{\tilde{C}}(s, \tilde{k})\right] p_{B}(s) d s\right\}
\end{gathered}
$$

we get

$$
\begin{gathered}
\frac{\hat{\delta}(\epsilon)-\delta(\epsilon)}{\lambda\left[\tilde{S}^{*} \cap\left[s^{\prime}-\epsilon, s^{\prime}+\epsilon\right]\right]}=\left\{\frac{K}{\int_{\hat{S}^{*} \backslash\left[s^{\prime}-\epsilon, s^{\prime}+\epsilon\right]} u^{\prime}\left[\hat{\nu}_{\tilde{C}}(s, \tilde{k})\right] p_{B}(s) d s}-\frac{H}{P\left[\hat{S}^{*} \backslash\left[s^{\prime}-\epsilon, s^{\prime}+\epsilon\right]\right]}\right\}- \\
\frac{1}{\int_{\hat{S}^{*} \backslash\left[s^{\prime}-\epsilon, s^{\prime}+\epsilon\right]} u^{\prime}\left[\hat{\nu}_{\tilde{C}}(s, \tilde{k})\right] p_{B}(s) d s} \frac{\hat{\delta}(\epsilon)}{\lambda\left[\tilde{S}^{*} \cap\left[s^{\prime}-\epsilon, s^{\prime}+\epsilon\right]\right]} \\
\left\{\frac{\int_{\hat{S}^{*} \backslash\left[s^{\prime}-\epsilon, s^{\prime}+\epsilon\right]}\left[u\left[\hat{\nu}_{\tilde{C}}(s, \tilde{k})+\hat{\delta}(\epsilon)\right]-u\left[\hat{\nu}_{\tilde{C}}(s, \tilde{k})\right]\right] p_{B}(s) d s}{\hat{\delta}(\epsilon)}-\int_{\hat{S}^{*} \backslash\left[s^{\prime}-\epsilon, s^{\prime}+\epsilon\right]} u^{\prime}\left[\hat{\nu}_{\tilde{C}}(s, \tilde{k})\right] p_{B}(s) d s\right\}
\end{gathered}
$$


Since the expression in the last line, tends to 0 as $\epsilon \rightarrow 0$ and thus $\hat{\delta}(\epsilon)$ tends to 0 , to show that the total expression is eventually negative, we need only show that as $\epsilon \rightarrow 0, \frac{\hat{\delta}(\epsilon)}{\lambda\left[\tilde{S}^{*} \cap\left[s^{\prime}-\epsilon, s^{\prime}+\epsilon\right]\right]}$ stays bounded. However, this follows since we have

$$
\frac{\hat{\delta}(\epsilon)}{2} \int_{\hat{S}^{*}} u^{\prime}\left[\hat{\nu}_{\tilde{C}}(s, \tilde{k})\right] p_{B}(s) d s<\int_{\hat{S}^{*} \backslash\left[s^{\prime}-\epsilon, s^{\prime}+\epsilon\right]}\left[u\left[\hat{\nu}_{\tilde{C}}(s, \tilde{k})+\hat{\delta}(\epsilon)\right]-u\left[\hat{\nu}_{\tilde{C}}(s, \tilde{k})\right]\right] p_{B}(s) d s
$$

for $\epsilon$ small i.e.

$$
\frac{\hat{\delta}(\epsilon)}{2} \int_{\hat{S}^{*}} u^{\prime}\left[\hat{\nu}_{\tilde{C}}(s, \tilde{k})\right] p_{B}(s) d s<K \lambda\left[\tilde{S}^{*} \cap\left[s^{\prime}-\epsilon, s^{\prime}+\epsilon\right]\right]
$$

so that

$$
\frac{\hat{\delta}(\epsilon)}{\lambda\left[\tilde{S}^{*} \cap\left[s^{\prime}-\epsilon, s^{\prime}+\epsilon\right]\right]}<\frac{K}{1 / 2 \int_{\hat{S}^{*}} u^{\prime}\left[\hat{\nu}_{\tilde{C}}(s, \tilde{k})\right] p_{B}(s) d s}
$$

for $\epsilon$ small. Thus we have arrived at the desired contradiction.

Remark: Note that if $\hat{\nu}_{\tilde{C}}(\hat{s}, \tilde{k})=F(\hat{s}, \tilde{C})-\tilde{R}$ the inequality in (16) cannot hold in a neighborhood of $\hat{s}$.

The proof of the other inequality, (17) is essentially identical: Suppose we had $>0$ in (17) and let $s^{\prime} \in \operatorname{int} \bar{S} \cap\left(S \backslash \tilde{S}^{*}\right)$ and assume that $P\left(\left(\bar{S} \backslash \tilde{S}^{*}\right) \cap\left[s^{\prime}-\epsilon, s^{\prime}+\epsilon\right]\right)>0, \forall \epsilon>0$. First, on $\left(\bar{S} \backslash \tilde{S}^{*}\right) \cap\left[s^{\prime}-\epsilon, s^{\prime}+\epsilon\right]$, change the payment to the entrepreneur from $F(s, \tilde{C})-\tilde{R}$ to $\hat{\nu}_{\tilde{C}}(s, \tilde{k})$ and add this set to the set of observations. Next, on $\hat{S}^{*}$, change the payment to the entrepreneur to $\hat{\nu}_{\tilde{C}}(s, \tilde{k})-\hat{\delta}(\epsilon)$ and let the lender receive $\delta(\epsilon)$ more. Due to continuity of $\hat{\nu}_{\tilde{C}}(\cdot, \tilde{k})$ and $F$ and compactness of $I$ and $\left[s^{\prime}-\epsilon, s^{\prime}+\epsilon\right]$, for small $\hat{\delta}$ incentive compatibility continues to hold. $\hat{\delta}$ and $\delta$ are defined as follows:

Let for $\bar{\epsilon}>0, \bar{I}=\left[s^{\prime}-\bar{\epsilon}, s+\bar{\epsilon}\right]$ s.t. $\forall s, \hat{s} \in \bar{I}$ :

$$
\frac{\left\{u\left[\hat{\nu}_{\tilde{C}}(s, \tilde{k})\right]-u[F(s, \tilde{k})-\tilde{R}]\right\}}{\left\{\hat{\nu}_{\tilde{C}}(\hat{s}, \tilde{k})-[F(\hat{s}, \tilde{k})-\tilde{R}]+c(\hat{s}, \tilde{C})\right\}} \frac{p_{B}(s)}{p(\hat{s})}-\frac{\int_{\hat{S}^{*}} u^{\prime}[\hat{\nu}(s, \tilde{k})] P_{B}(d s)}{P\left[\hat{S}^{*}\right]}>0
$$

Let

$$
H=\max _{s \in \bar{I}}\left\{\hat{\nu}_{\tilde{C}}(s, \tilde{k})-F[(s, \tilde{C})-\tilde{R}]+c(s, \tilde{C})\right\} p(s)
$$

and define for sufficiently small $\bar{\epsilon}>\epsilon>0, \delta(\epsilon)$ by:

$$
-\int_{\left(\bar{S} \backslash \tilde{S}^{*}\right) \cap\left[s^{\prime}-\epsilon, s^{\prime}+\epsilon\right]} H d s+\int_{\hat{S}^{*}} \delta(\epsilon) p(s) d s=0
$$

i.e.

$$
\delta(\epsilon)=\frac{H \lambda\left[\left(\bar{S} \backslash \tilde{S}^{*}\right) \cap\left[s^{\prime}-\epsilon, s^{\prime}+\epsilon\right]\right]}{P\left(\hat{S}^{*}\right)}
$$

Let

$$
K=\min _{s \in \bar{I}}\left\{u\left[\hat{\nu}_{\tilde{C}}\right]-u[F(s, \tilde{C})-\tilde{R}]\right\} p_{B}(s)
$$


and (for sufficiently small $\bar{\epsilon}>\epsilon>0$ ) let $\hat{\delta}(\epsilon)$ be defined by:

$$
\int_{\left(\bar{S} \backslash \tilde{S}^{*}\right) \cap\left[s^{\prime}-\epsilon, s^{\prime}+\epsilon\right]} K d s+\int_{\hat{S}^{*}}\left\{u\left[\hat{\nu}_{\tilde{C}}(s, \tilde{k})-\hat{\delta}(\epsilon)\right]-u\left[\hat{\nu}_{\tilde{C}}(s, \tilde{k})\right]\right\} p_{B}(s) d s=0
$$

Both parties are then not worse off with these changes and as before we need to show feasibility, i.e. that there is $\epsilon>0$ s.t. $\hat{\delta}(\epsilon)>\delta(\epsilon)$.

Similarly to before we get

$$
\begin{gathered}
\frac{\hat{\delta}(\epsilon)-\delta(\epsilon)}{\lambda\left[\left(\bar{S} \backslash \tilde{S}^{*}\right) \cap\left[s^{\prime}-\epsilon, s^{\prime}+\epsilon\right]\right]}=\left\{\frac{K}{\int_{\hat{S}^{*}} u^{\prime}\left[\hat{\nu}_{\tilde{C}}(s, \tilde{k})\right] p_{B}(s) d s}-\frac{H}{P\left(\hat{S}^{*}\right)}\right\}- \\
\frac{1}{\int_{\hat{S}^{*}} u^{\prime}\left[\hat{\nu}_{\tilde{C}}(s, \tilde{k})\right] p_{B}(s) d s} \frac{\hat{\delta}(\epsilon)}{\lambda\left[\left(\bar{S} \backslash \tilde{S}^{*}\right) \cap\left[s^{\prime}-\epsilon, s^{\prime}+\epsilon\right]\right]} \\
\left\{\frac{\left.\int_{\hat{S}^{*}\left[u\left[\hat{\nu}_{\tilde{C}}(s, \tilde{k})-\hat{\delta}(\epsilon)\right]-u\left[\hat{\nu}_{\tilde{C}}(s, \tilde{k})\right]\right] p_{B}(s) d s}^{\hat{\delta}(\epsilon)}-\int_{\hat{S}^{*}} u^{\prime}\left[\hat{\nu}_{\tilde{C}}(s, \tilde{k})\right] p_{B}(s) d s\right\}}{}\right.
\end{gathered}
$$

which is now $>0$ for small $\epsilon$, where again we use that $\frac{\hat{\delta}(\epsilon)}{\lambda\left[\left(\bar{S} \backslash \tilde{S}^{*}\right) \cap\left[s^{\prime}-\epsilon, s^{\prime}+\epsilon\right]\right]}$ stays bounded as $\epsilon \rightarrow 0$

The interpretation of (16) and (17) is relatively simple and follows from the manner in which they were derived. The first term in (16) describes the gain in expected utility per unit of extra expected money spent on including the state $s^{\prime}$ in the set of observation. The second term quantifies the gain in expected utility per unit of expected money spent on increasing the payment to the entrepreneur marginally on the set of observation. In a state of observation the first minus the second has to be positive. In a state of non-observation, it has to be negative (else that state would have to be included in $\tilde{S}^{*}$ ). The gap between the return of the entrepreneur in the observation set and outside it, present at the boundaries of these sets can be explained as follows. Suppose we had $\hat{\nu}_{\tilde{C}}\left(s^{\prime}, \tilde{k}\right)=F\left(s^{\prime}, \tilde{C}\right)-R$ and $s^{\prime} \in \tilde{S}^{*}$. If we make $\tilde{S}^{*}$ smaller we save a positive observation cost, while the change in utility of the entrepreneur is small. Consequently, this change is feasible and obviously welfare increasing (because observation costs are reduced).

Under the assumptions stated in the proposition we have a corollary which summarizes the optimal contract over the entire state space (retaining $\tilde{\nu}$ as being the payment to the borrower in any state $s$ ).

\section{Corollary 1}

For an optimal contract, $\left(\tilde{C}, \tilde{k}, \tilde{S}^{*}, \tilde{R}\right)$ with $P\left(\tilde{S}^{*}\right)>0$ and $P\left(S \backslash \tilde{S}^{*}\right)>0$, we have:

(i) $\tilde{\nu}(s)=\hat{\nu}_{\tilde{C}}(s, \tilde{k})$ and $s \in \tilde{S}^{*}$ for $P$-a.a. $s$ satisfying either

(I) $F(s, \tilde{C})-\tilde{R}<0$;

or 
(a) $\quad \hat{\nu}_{\tilde{C}}(s, \tilde{k})>F(s, \tilde{C})-\tilde{R} \geq 0$;

and

(b) $\quad \frac{u\left[\hat{\nu}_{\tilde{C}}(s, \tilde{k})\right]-u[F(s, \tilde{C})-\tilde{R}]}{\hat{\nu}_{\tilde{C}}(s, \tilde{k})-[F(s, \tilde{C})-\tilde{R}]+c(s, \tilde{C})}>u^{\prime}\left[\hat{\nu}_{\tilde{C}}(s, \tilde{k}]\right.$

(ii) $\tilde{\nu}(s)=F(s, \tilde{C})-\tilde{R}$ and $s \in S \backslash \tilde{S}^{*}$ for P-a.a. $s$ satisfying either:

(I)

(a) $\quad \hat{\nu}_{\tilde{C}}(s, \tilde{k})>F(s, \tilde{C})-\tilde{R} \geq 0$;

and

(b) $\quad \frac{u\left[\hat{\nu}_{\tilde{C}}(s, \tilde{k})\right]-u[F(s, \tilde{C})-\tilde{R}]}{\hat{\nu}_{\tilde{C}}(s, \tilde{k})-[F(s, \tilde{C})-\tilde{R}]+c(s, \tilde{C})}<u^{\prime}\left[\hat{\nu}_{\tilde{C}}(s, \tilde{k}]\right.$

or

(II) $\hat{\nu}_{\tilde{C}}(s, \tilde{k}) \leq F(s, \tilde{C})-\tilde{R}$;

Proof: For $s, s^{\prime}$ such that $\hat{\nu}_{\tilde{C}}(s, \tilde{k})>0, \hat{\nu}_{\tilde{C}}\left(s^{\prime}, \tilde{k}\right)>0, p\left(s^{\prime}\right)>0$ we have by $(15)$ :

$$
u^{\prime}\left[\hat{\nu}_{\tilde{C}}(s, \tilde{k})\right] p_{B}(s)=p(s) u^{\prime}\left[\hat{\nu}_{\tilde{C}}\left(s^{\prime}, \tilde{k}\right)\right] \frac{p_{B}\left(s^{\prime}\right)}{p\left(s^{\prime}\right)}
$$

Thus

$$
\frac{\int_{\hat{S}^{*}} u^{\prime}\left[\hat{\nu}_{\tilde{C}}(s, \tilde{k})\right] P_{B}(d s)}{P\left[\hat{S}^{*}\right]}=u^{\prime}\left[\hat{\nu}_{\tilde{C}}(s, \tilde{k})\right] \frac{p_{B}\left(s^{\prime}\right)}{p\left(s^{\prime}\right)}
$$

The Corollary then follows from the discussion before Proposition 1 and the Proposition itself

\section{Further Characterization of the Optimal Contract}

Suppose now that the set of $s$ s.t.

$$
\frac{u\left[\hat{\nu}_{\tilde{C}}(s, \tilde{k})\right]-u[F(s, \tilde{C})-\tilde{R}]}{\hat{\nu}_{\tilde{C}}(s, \tilde{k})-[F(s, \tilde{C})-\tilde{R}]+c(s, \tilde{C})}=u^{\prime}\left[\hat{\nu}_{\tilde{C}}(s, \tilde{k}]\right.
$$

has P-measure 0. Then the previous Corollary provides a complete characterization of the optimal contract.

\section{Corollary 2}

Suppose the set where (18) holds has measure 0 . Then $\tilde{S}^{*}$ is either empty or a union of intervals: $\tilde{S}^{*}=\cup_{n=0}^{N} I_{n}$, where $N \leq \infty$. Furthermore, for any boundary point, $s$ of $\tilde{S}^{*}$ s.t. $\hat{\nu}_{\tilde{C}}(s, \tilde{k})>0$, we have $\hat{\nu}_{\tilde{C}}(s, \tilde{k})>F(\tilde{S}, \tilde{C})-\tilde{R}$.

Proof: The set where $F(s, \tilde{C})-\tilde{R} \leq 0$ is, unless empty, by assumption A.6 a closed interval. If not empty, the set where $\hat{\nu}_{\tilde{C}}(s, \tilde{k})>F(s, \tilde{C})-\tilde{R} \geq 0$ and $\frac{u\left[\hat{\nu}_{\tilde{C}}(s, \tilde{k})\right]-u[F(s, \tilde{C})-\tilde{R}]}{\hat{\nu}_{\tilde{C}}(s, \tilde{k})-[F(s, \tilde{C})-\tilde{R}]+c(s, \tilde{C})}>u^{\prime}\left[\hat{\nu}_{\tilde{C}}(s, \tilde{k}]\right.$ is, by continuity, a union of intervals. 
Suppose now that $s^{\prime}$ is a boundary point for $\tilde{S}^{*}$ and that $\hat{\nu}_{\tilde{C}}\left(s^{\prime}, \tilde{k}\right)>0$. If $F\left(s^{\prime}, \tilde{C}\right)-\tilde{R}=0$ the desired inequality holds. Else, we have $\geq$ in $(18)$ and hence $\hat{\nu}_{\tilde{C}}(s, \tilde{k})>F(s, \tilde{C})-\tilde{R}$

Note that the Corollary says (under the stated assumptions) that on any bounded interval, $I$ in $\tilde{S}^{*}$ there is $\epsilon>0$ s.t. $\hat{\nu}_{\tilde{C}}(s, \tilde{k})-\left[F\left(\tilde{s}_{0}, \tilde{C}\right)-\tilde{R}\right]>\epsilon$ for $s \in I$. The incentive compatibility constraint is thus always slack (in a strong sense) when $\hat{\nu}_{\tilde{C}}(s, \tilde{k})>0 P$-a.s. which is certainly the case when $P_{B}=P$ (because of risk aversion) and thus also the case when $P_{B}$ and $P$ are not too different.

This is similar to a statement in Garino and $\operatorname{Simmons}(2006)$ that the incentive compatibility constraint is slack. However, we should note that their model is not exactly identical to ours since (i) the ability of the entrepreneur to repay in our case may depend on the investment in the project, $C+N=B$ and (ii) the observation costs in our model may depend on the state, while it is independent of the state in Garino and Simmons. ${ }^{6}$

\subsection{When is $\tilde{S}^{*}$ an Interval?}

Exploring Corollary 1, we now address the question of when is $\tilde{S}^{*}$ an interval? Note first that if $F(s, \tilde{C})-\tilde{R} \geq \hat{\nu}_{\tilde{C}}(s, \tilde{k}), \forall s, \tilde{S}^{*}$ is empty and there is never any observation. On the other hand, if $F(s, \tilde{R})-\tilde{R}<0, \forall s \in S, \tilde{S}^{*}=S$. Finally, if $0 \leq F(s, \tilde{C})-\tilde{R} \leq \hat{\nu}_{\tilde{C}}(s, \tilde{k}), \forall s$ whether the contract is trivial $\left(\tilde{S}^{*}=\emptyset\right.$ or $\tilde{S}^{*}=S$ ) depends on whether there is a solution to (18) or not.

\section{Corollary 3}

Suppose that $S_{0}$ is an interval or empty, that $S_{1}$ is an interval of the form $\left[s_{1}, \infty\right)$ and that there is at most one point, $s^{*}<s_{1}$ where (18) holds. Then $\tilde{S}^{*}$ is an interval or empty.

Proof: $\tilde{S}^{*} \subset\left[0, s_{1}\right)$ and at $s_{1}$ (where by continuity, $\hat{\nu}_{C}\left(s_{1}, \tilde{k}\right)=F\left(s_{1}, \tilde{C}\right)-R$ ) we have $<$ in (18). Let $s_{0}=\max S_{0}$ if $S_{0}$ is non-empty, 0, else. If at $s_{0}$ we have $>$ in (18), then if $s^{*}$ exists, $s^{*} \in\left[s_{0}, s_{1}\right]$ and $\tilde{S}^{*}=\left[0, s^{*}\right]$. Else, $\tilde{S}^{*}=\emptyset$ or equal to $\left[0, s_{0}\right]$

From this corollary immediately follows

\footnotetext{
${ }^{6}$ The analysis in Garino and $\operatorname{Simmons}(2006)$ that leads to their conclusion is not formally correct. In the appendix, A.3.4 (p. 104) they claim to demonstrate that it is not possible to have the payment to the entrepreneur equal to 0 in states of observations and to have $s^{*}=R$. This claim is based on an analysis of the function$$
R_{0}^{U}\left(s^{*}\right)=\frac{(1+i) L+c H\left(s^{*}\right)-\int_{0}^{s^{*}} s H(d s)}{1-H\left(s^{*}\right)}
$$

where $H$ is the distribution function for the state variable $s$ and $L$ is the size of the loan. Also (equation (1), p. 77) they assume $\int_{S} s H(d s)-c>L(1+i)$. It follows that $R_{0}^{U}(0)=(1+i) L>0$ and as $s \rightarrow \sup S, R_{0}^{U}(s) \rightarrow-\infty$. As a consequence, there is at least one $s^{*}$ s.t. $R_{0}^{U}\left(s^{*}\right)=s^{*}$, contrary to their claim that $R_{0}^{U}\left(s^{*}\right)$ is bounded above by $s^{*}-c$.
} 


\section{Corollary 4}

The following two conditions together imply that $\tilde{S}^{*}$ is an interval if it is $\neq \emptyset$.

A.9 $\hat{\nu}_{\tilde{C}}(\cdot, \tilde{k})$ cuts $F(\cdot, \tilde{C})-\tilde{R}$ once from above, in $\hat{s}(\tilde{C}, \tilde{k}, \tilde{R})$.

A.10 $\forall \tilde{k}, \tilde{C}$ and $\tilde{R}$ there is at most one $s=s^{*}<\hat{s}(\tilde{C}, \tilde{k}, \tilde{R})$ s.t. (18) holds

From Corollary 4 in turn follows

\section{Corollary 5}

Suppose that $\hat{\nu}_{\tilde{C}}(\cdot, \tilde{k})$ is constant, $=\kappa>0$ and that in any $s$ either $c(\cdot, \tilde{C})$ or $F(\cdot, \tilde{C})$ is strictly increasing. Then $\tilde{S}^{*}$ is an interval or empty.

Proof: Suppose $\exists s^{*}$ s.t. $\kappa>F\left(s^{*}, \tilde{C}\right)-\tilde{R} \geq 0$ and (18) holds, that is

$$
u[\kappa]-u\left[F\left(s^{*}, \tilde{C}\right)-\tilde{R}\right]-u^{\prime}[\kappa]\left\{\kappa-\left[F\left(s^{*}, \tilde{C}\right)-\tilde{R}\right]\right\}-u^{\prime}[\kappa] c\left(s^{*}, \tilde{C}\right)=0
$$

If as we increase $s, F(s, \tilde{C})$ increases, then because of the strict concavity of $u, u[\kappa]-u[F(s, \tilde{C})-$ $\tilde{R}]-u^{\prime}[\kappa]\{\kappa-[F(s, \tilde{C})-\tilde{R}]\}$ decreases. If $c(s, \tilde{C})$ increases in $s,-u^{\prime}[\kappa] c(s, \tilde{C})$ decreases in $s$. It follows that the left hand side is decreasing in $s$, hence $s^{*}$ is unique.

The two condition of Corollary 4 are then fulfilled

Note that when $P_{B}$ and $P$ are close to being equal and when the conditions of Corollary 5 hold, $\tilde{S}^{*}$ will continue to be an interval.

We can rewrite (18) as:

$$
\frac{\frac{u\left[\hat{\nu}_{C}(s, k)\right]-u[F(s, C)-R]}{\hat{\nu}_{C}(s, k)-[F(s, C)-R]} \frac{1}{u^{\prime}\left[\hat{\nu}_{C}(s, k)\right]}}{1+\frac{c(s, C)}{\hat{\nu}_{C}(s, k)-[F(s, C)-R]}}=1
$$

The following two sets of assumptions each imply that (19) has, for any $k, C$, and $R$, at most a single solution on the set where $\hat{\nu}_{\tilde{C}}(\cdot, \tilde{k})>F(\cdot, \tilde{C})-\tilde{R}$ :

A.11 (i) $\hat{\nu}_{C}(s, k)-[F(s, C)-R]$ is, for all $C R$, and $k$ non-increasing in $s$;

A.11 (ii) $c(s, C)$ is, for all $C$, non-decreasing in $s$;

A.11 (iii) For all $C, k$, and $R$,

$$
\frac{u\left[\hat{\nu}_{C}(s, k)\right]-u[F(s, C)-R]}{\hat{\nu}_{C}(s, k)-[F(s, C)-R]} \frac{1}{u^{\prime}\left[\hat{\nu}_{C}(s, k)\right]} \text { is strictly decreasing in } s
$$

A.12 (i) $\hat{\nu}_{C}(s, k)-[F(s, C)-R]$ is, for all $C$ and $R$ non-increasing in $s$;

A.12 (ii) $c(s, C)$ is, for all $C$, strictly increasing in $s$;

A.12 (iii) For all $C, k$, and $R$,

$$
\frac{u\left[\hat{\nu}_{C}(s, k)\right]-u[F(s, C)-R]}{\hat{\nu}_{C}(s, k)-[F(s, C)-R]} \frac{1}{u^{\prime}\left[\hat{\nu}_{C}(s, k)\right]} \text { is non-increasing in } s
$$


To explain how we may arrive at A.11 (iii) or A.12 (iii) when we know that $\hat{\nu}_{C}(s, k)$ is strictly increasing in $s, \forall C, k$ (a case which has our special interest), we may exploit the identity

$$
\frac{d}{d x}\left[\frac{u(x)-u(x-\Delta(x))}{\Delta(x) u^{\prime}(x)}\right]=\frac{d \Delta}{d x} \frac{\partial}{\partial \Delta}\left[\frac{u(x)-u(x-\Delta)}{\Delta u^{\prime}(x)}\right]+\frac{\partial}{\partial x}\left[\frac{u(x)-u(x-\Delta)}{\Delta u^{\prime}(x)}\right]
$$

By Assumption A.11 (i) or A.12 (i),$\frac{d \Delta}{d x} \leq 0$. Since $u$ is strictly concave, we have

$$
\frac{\partial}{\partial \Delta}\left[\frac{u(x)-u(x-\Delta)}{\Delta u^{\prime}(x)}\right]=\frac{u^{\prime}(x-\Delta)-\frac{u(x)-u(x-\Delta)}{\Delta}}{\Delta u^{\prime}(x)}>0
$$

Thus if

$$
\text { for } x>\Delta>0, \quad \frac{\partial}{\partial x}\left[\frac{u(x)-u(x-\Delta)}{\Delta u^{\prime}(x)}\right]<0
$$

then A.11 (iii) holds. If (21) holds with weak inequality, A12 (iii) holds.

\section{Examples of (21)}

(i) $u(x)=x^{a}, 0<a<1$

Then

$\left[u^{\prime}(x)-u^{\prime}(x-\Delta)\right] u^{\prime}(x)-u^{\prime \prime}(x)[u(x)-u(x-\Delta)]=$

$\left[a x^{a-1}-a(x-\Delta)^{a-1}\right] a x^{a-1}-a(a-1) x^{a-2}\left[x^{a}-(x-\Delta)^{a}\right]=$

$-a^{2}[x-\Delta]^{a-1} x^{a-1}+a x^{2(a-1)}+a(a-1) x^{a-2}(x-\Delta)^{a}$

which equals 0 , when $\Delta=0$. Next differentiating this expression w.r.t. $\Delta$ we get

$a^{2}(a-1)[x-\Delta]^{a-2} x^{a-1}-a^{2}(a-1) x^{a-2}(x-\Delta)^{a-1}=x^{a-1}\left[a^{2}(a-1)\right]\left[(x-\Delta)^{a-2}-x^{-1}(x-\Delta)^{a-1}\right]<0$, when $0<\Delta<x$.

Since $a<1$ and $0<x-\Delta<x$, it follows that (21) holds.

(ii) $u(x)=-e^{-a x}, a>0$

In this case

$\left[u^{\prime}(x)-u^{\prime}(x-\Delta)\right] u^{\prime}(x)-u^{\prime \prime}(x)[u(x)-u(x-\Delta)]=\left[a e^{-a x}-a e^{-a(x-\Delta)}\right] a e^{-a x}+a^{2} e^{-a x}\left[-e^{-a x}+\right.$ $\left.e^{-a(x-\Delta)}\right]=0$

and (21) holds with weak inequality.

\subsection{Conditions on Beliefs}

We are interested in the case when the borrower is "optimistic" relative to the bank. We turn to defining this concept more precisely.

Suppose that the density, $p$ of $P$ is positive on $[0, \bar{s}]$, where possibly $\bar{s}=\infty$ and that the Radon-Nikodym derivative $\frac{p_{B}}{p}$ is differentiable. ${ }^{7}$ We shall explore the following condition:

$$
\forall s \in S: \frac{\mathrm{d}}{\mathrm{d} s}\left(\frac{p_{B}(s)}{p(s)}\right) \geq 0
$$

\footnotetext{
${ }^{7}$ The derivative is called the likelihood ratio by some.
} 
If the Radon-Nikodym derivative, $\frac{p_{B}(s)}{p(s)}$ is not differentiable, an equivalent condition to $(22)$ is:

$$
\forall \hat{s} \in(0, \bar{s}]: \frac{P_{B}(s, \hat{s}]}{P[s, \hat{s}]} \text { is non-decreasing in } s<\hat{s}
$$

To see the relationship between the two conditions, consider the following

\section{Lemma 3}

Suppose $f$ is continuous and (weakly) increasing. Then

$$
\forall \tilde{s}<\hat{s}<\bar{s}: \frac{\mathrm{d}}{\mathrm{d} \tilde{s}} \frac{\int_{\tilde{s}}^{\hat{s}} f(s) p(s) d s}{\int_{\tilde{s}}^{\hat{s}} p(s) d s} \geq 0
$$

If on the other hand (24) holds, then $f$ is (weakly) increasing.

Proof: Writing out the derivative in (24) we get:

$$
\frac{-f(\tilde{s}) p(\tilde{s}) \int_{\tilde{s}}^{\hat{s}} p(s) d s+p(\tilde{s}) \int_{\tilde{s}}^{\hat{s}} f(s) p(s) d s}{P[\tilde{s}, \hat{s}]^{2}}=\frac{p(\tilde{s})}{P[\tilde{s}, \hat{s}]^{2}} \int_{\tilde{s}}^{\hat{s}}[f(s)-f(\tilde{s})] p(s) d s \geq 0
$$

On the other hand, if $f$ is not (weakly) increasing there is $\tilde{s}<\hat{s}$, s.t. on $(\tilde{s}, \hat{s}), f$ is strictly decreasing. In that case, the expression $\int_{\tilde{s}}^{\hat{s}}[f(s)-f(\tilde{s})] p(s) d s$ in the previous equation is $<0$

To interpret (22), observe that the condition (23) implies that for all $s, P_{B}(s, 1] \geq P(s, 1]$, with strict inequality if $P_{B} \neq P$, in other words that the project as viewed by the entrepreneur first order stochastically dominates the project as viewed by the bank ${ }^{8}$. We thus interpret (22) to express the relative optimism of the borrower.

The first order condition for $\hat{\nu}_{C}(\cdot, k)$ can be stated as:

$$
u\left[\hat{\nu}_{C}(s, k)\right]=\frac{p(s)}{p_{B}(s)} u^{\prime}\left[\hat{\nu}_{C}\left(s^{\prime}, k\right)\right] \frac{p_{B}\left(s^{\prime}\right)}{p\left(s^{\prime}\right)}
$$

for $P$-a.a. $s, s^{\prime}$.

If $\frac{p(s)}{p_{B}(s)}$ is differentiable, then $(22)$ is equivalent to $\hat{\nu}_{C}(\cdot, k)$ being differentiable with a derivative, $\frac{\partial}{\partial s} \hat{\nu}_{C}(s, k)>0$ which is continuous $P$-a.s. One more implication of $(22)$ and $(25)$ is that $\hat{\nu}_{C}(\cdot, k)$ is strictly concave in $s$ if two further conditions are satisfied: (i) $u^{\prime \prime \prime}>0$ and (ii) $\frac{p(s)}{p_{B}(s)}$ is concave in $s$.

Some Further Examples

(i) Suppose $u(x)=-e^{-a x}$ and that both the bank and the borrower use exponential distribution. We then have that $\frac{p_{B}(s)}{p(s)}=\frac{\lambda_{B}}{\lambda} e^{\left(\lambda-\lambda_{B}\right) s}$ and if $\lambda>\lambda_{B}$, indeed $\frac{\mathrm{d}}{\mathrm{d}} \frac{p_{B}(s)}{p(s)}>0$. In this case $\hat{\nu}_{C}(\cdot, k)$ is

\footnotetext{
${ }^{8}$ It is not difficult to see that our condition is stronger than "first order stochastic dominance".
} 
affine on its positive segment: $\hat{\nu}_{C}(s, k)=\max \left\{0, b+\frac{\lambda-\lambda_{B}}{a} s\right\}$. To see this, check that the first order conditions hold:

$$
a e^{-a\left[b+\frac{\lambda-\lambda_{B}}{a} s\right]} \frac{\lambda_{B}}{\lambda} e^{\left(\lambda-\lambda_{B}\right) s}=\frac{\lambda_{B}}{\lambda} a e^{-a b}, \forall s
$$

If $F(s, C)=f(C) s$ with $f(0)=0, f^{\prime}>0$ and $c(s, C)$ is non-decreasing in $s$ then for an optimal contract, the set of observations, $\tilde{S}^{*}$ will always be an interval if $\tilde{B}>0$ and $b>0$. If $f(\tilde{C})>\frac{\lambda-\lambda_{B}}{a}$ then $\hat{\nu}_{\tilde{C}}(\cdot, \tilde{k})-F(\cdot, \tilde{C})$ is decreasing in $s$ and $\tilde{S}^{*}$ will be a compact interval. If $f(\tilde{C})<\frac{\lambda-\lambda_{B}}{a}$ then $\tilde{S}^{*}=\Re_{+}$is possible, that is all states are observed.

In the next example we develop a density that leads to $\hat{\nu}_{C}(\cdot, k)$ being increasing and strictly concave.

(ii) Suppose $u(x)=x^{a}, 0<a<1$ and that $\frac{p_{B}(s)}{p(s)}=D s^{b}$ on $[0, \bar{s}], 0$ else, with $b>0$. In that case the first order condition for $\hat{\nu}_{C}$ is

$$
a\left[\hat{\nu}_{C}(s, k)\right]^{a-1} D s^{b}=a\left[\hat{\nu}_{C}\left(s^{\prime}, k\right)\right]^{a-1} D s^{\prime b}, \forall s
$$

so, $\hat{\nu}_{C}(s, k)=g(k) s^{\frac{b}{1-a}}$ for some function, $g$ and

$$
\frac{d}{d s} \hat{\nu}_{C}(s, k)=\frac{b}{1-a} g(k) s^{\frac{b}{1-a}-1}>0
$$

If $\frac{b}{1-a}<1$ we have $\frac{d^{2}}{d s^{2}} \hat{\nu}_{C}(s, k)<0$, i.e. strict concavity.

The densities $p_{B}$ and $p$ that lead to the relation $\frac{p_{B}(s)}{p(s)}=D s^{b}$ could be as follows:

$$
p_{B}(s)=\frac{1-\beta}{\bar{s}^{1-\beta}} s^{-\beta}, p(s)=\frac{1-\alpha}{\bar{s}^{1-\alpha}} s^{-\alpha} \text { and then } b=\alpha-\beta \text { and } D=\frac{(1-\beta) \bar{s}^{1-\alpha}}{(1-\alpha) \bar{s}^{1-\beta}} \text {. }
$$

\section{Concluding Remarks}

We have studied the form of the optimal financial contract under costly state verification making the natural assumptions of a risk averse borrower and diverse subjective expectations. In this more general setting the contract tends to become more complex since it has to be sensitive to both preferences and beliefs. When information is asymmetric resulting in incomplete markets, financial contracts act as substitutes for markets and under such conditions a financial contracts may have to serve multiple purposes. In our case the contract is not used only for the transfer of money between periods. It also provides an insurance vehicle and, in addition, permits agents to trade on their different assessments of the likelihood of future states of the world. Such multi faceted contracts clearly are what we find in today's financial markets. Of course, all the theoretical possibilities allowed in our abstract framework may not have exact real counterparts, since some features of contracts found in real markets depend not only on preferences and beliefs but also on the institutional setting. Moreover, real contracts may also depend on other types of asymmetric information not considered here. 
In the final part of the paper we have provided conditions under which the optimal contract retains one feature which is often found in reality, namely that the region of observations (which can in that case be interpreted as a region of non-performance or bankruptcy) is a single interval consisting of low-outcome states. Such a contract has the important feature that the pay-off to the entrepreneur varies with the observed state. We have explained that this feature is often found in observed contracts. In our setting this conclusion flows directly from the nature of diverse beliefs.

\section{References}

Atkeson, A. and R.E. Lucas, jr.(1992): "On Efficient Distribution with Private Information." Review of Economic Studies 59, 427-453.

Bernanke, B.S., and M. Gertler (1989): "Agency Costs, Net Worth, and Business Fluctuations", American Economic Review, 79, 14-31

Bernanke, B.S., M. Gertler and S. Gilchrist (1999): "The Financial Accelerator in a Quantitative Business Cycle Framework." In J. Taylor and M. Woodford (eds.) The Handbook of Macroeconomics, Volume IC. Amsterdam: North-Holland, Chapter 21, 1342-1385.

Boyd, J.H. and B.D. Smith (1994): "How Good are Standard Debt Contracts? Stochastic vs. Nonstochastic Monitoring in a Costly State Verification Environment." Journal of Business, 67, 539-561.

Cole, H.L. and N.R. Kocherlakota (2001): "Efficient Allocation with Hidden Income and Hidden Storage." Review of Economic Studies, 68, 523-542.

Carlier, G., and L. Renou: "A Caostly State Verification Model with Diversity of Opinions". Economic Theory, 25, 497-504.

Carlstrom, C.T., and T.S. Fuerst (1997): "Agency Costs, Net Worth, and Business Fluctuations: A computable General Equilibrium Framework". American Economic Review, 87, 893-910.

Frankel, J.A. and K.A. Froot (1987): "Using Survey Data to Test Standard Propositions Regarding Exchange Rate Expectations." American Economic Review, 77, 133-153.

Frankel, J.A. and K.A. Froot (1990): "Chartists, Fundamentalists and the Demand for Dollars." In Courakis, A.S. and M.P. Taylor (eds.) Private Behavior and Government Policy in Interdependent Economies, Oxford University Press, New York, 73-126. 
Frankel, J.A. and A.K Rose (1995): "A Survey of Empirical Research on Nominal Exchange Rates." In Grossman, G.M. and K. Rogoff (ed) Handbook of International Economics, Vol. III, North-Holland, Amsterdam, 1689-1729.

Freixas, X. and R.C. Rochet (1997): Microeconomics of Banking MIT Press, Cambridge.

Gale, D. and M. Hellwig (1985): "Incentive-compatible Debt Contracts: The One-period Problem." Review of Economic Studies, 52, 647-663.

Garino, G. and P. Simmons (2006): "Costly State Verification with Varying Risk Preferences and Liability." Journal of Economic Surveys, 20, 71-109.

Innes, R.D. (1990): "Limited Liability and Incentive Contracting with Ex-ante Actin Choices." Journal of Economic Theory, 50, 45 - 67.

Kandel, E. and N.D. Pearson (1995): "Differential Interpretation of Public Signals and Trade in Speculative Markets." Journal of Political Economy, 4, 831 - 872.

Khahil, F. and B. Parigi (1998) : "Loan Size as Commitment." Rand Journal of Economics, 28, $629-640$.

Kurz, M. (2001): "Heterogenous Forecasting and Federal Reserve Information." Working Paper number 02-002, Department of Economics, Stanford University.

Mookherjee, D. and I. Png (1989): "Auditing, Insurance and Redistribution." Quarterly Journal of Economics, XX, 397-415.

Takagi, S. (1991): "Exchange Rate Expectations: A Survey of Survey Studies." International Monetary Fund Staff Papers 38, 156 - 183.

Romer, D. (2001): "Advanced Macroeconomics" McGraw Hill, Boston.

Townsend, R. (1979): "Optimal Contracts and Competitive Markets with Costly State Verification." Journal of Economic Theory, 21, 265-293. 